\title{
Effects of hydrogen blending on hydraulic and thermal characteristics of natural gas pipeline and pipe network
}

\author{
Heng Zhang ${ }^{1}$ (D), Jingfa $\mathrm{Li}^{1, *}$ (D), Yue $\mathrm{Su}^{2}$, Peng Wang ${ }^{1}$, and Bo Yu ${ }^{1}$ (D) \\ ${ }^{1}$ School of Mechanical Engineering, Beijing Key Laboratory of Pipeline Critical Technology and Equipment for Deepwater \\ Oil and Gas Development, Beijing Institute of Petrochemical Technology, Beijing 102617, PR China \\ ${ }^{2}$ Beijing Key Laboratory of Process Fluid Filtration and Separation, College of Mechanical and Transportation Engineering, China \\ University of Petroleum (Beijing), Beijing 102249, PR China
}

Received: 20 July 2021 / Accepted: 20 September 2021

\begin{abstract}
Blending a fraction of hydrogen into the natural gas pipeline or urban pipe network is an efficient approach for hydrogen delivery. In this paper, the mathematical model of Hydrogen-Blended Natural Gas (HBNG) transportation is established, and the influences of hydrogen blending on hydraulic and thermal characteristics of natural gas pipeline and pipe network are numerically investigated. The impact of hydrogen blending ratio on the performance of centrifugal compressor and the operating point for joint operation of pipeline and compressor is discussed. Results illustrate that compared with natural gas without hydrogen, the hydrogen blending can reduce the pipeline friction resistance and increase the volume flow rate. However, due to the lower volumetric calorific value of HBNG, the energy flow rate actually decreases under the same transportation condition. Meanwhile, the temperature drop along the pipeline slows down due to the blended hydrogen. The performance degradation of centrifugal compressor occurs with the increasing hydrogen blending ratio, and the operating point for joint operation of pipeline and centrifugal compressor moves to the direction of higher volume flow rate and lower pressure. This study is expected to shed a light on the hydrogen delivery by natural gas pipelines and pipe networks.
\end{abstract}

\section{Introduction}

Air pollution and global warming are two major environmental threats to human beings nowadays, which are tightly related to the overuse of fossil fuels. The development and utilization of low-carbon-intensity fuels (such as natural gas, hydrogen, etc.) are of great significance to the energy conservation, emission reduction and energy structure optimization [1-3]. As a kind of secondary energy, hydrogen has been recognized as a clean energy for its zerocarbon merit as only water is produced in its combustion. However, currently the utilization of hydrogen is heavily limited by the insufficient development of hydrogen transportation and distribution facilities. Pipeline transport is an economic and efficient way for long-distance and largescale natural gas transmission, and the relevant technologies have been maturely developed in past years. Literature studies have preliminarily shown that blending a certain amount of hydrogen into natural gas and using the existing natural gas pipelines or urban pipe networks for transportation is an efficient method for hydrogen delivery [4-7]. It is also noted that the transportation of hydrogen through

\footnotetext{
* Corresponding author: lijingfa@bipt.edu.cn
}

natural gas pipelines is a low-cost option and is beneficial for emission reduction [8-10]. Therefore, it is meaningful to study the transportation of hydrogen through natural gas pipelines and pipe networks.

Due to the substantial difference in physical and thermal properties of hydrogen and natural gas, the influences of hydrogen blending on hydraulic and thermal characteristics of natural gas pipeline and pipe network should be fully considered in the transportation technology. At present, researchers at home and abroad have devoted effort to exploring the effects of hydrogen blending on natural gas pipeline transmission [11-13]. For example, Tabkhi et al. [14] applied an isothermal model of natural gas pipeline transportation to investigate the impact of hydrogen blending on hydraulic conditions of a long-distance pipeline. It was found adding hydrogen into natural gas significantly reduces the pipeline transmission capacity, and the maximum allowable hydrogen blending ratio is about $6 \%$ (mass fraction) without changing any facilities in the pipeline system. Elaoud and Hadj-Taïeb [15] and Elaoud et al. [16] assessed the pressure variation of a small-scale ringed natural gas pipe network associated with hydrogen blending at steady and transient states by using isothermal models. Results showed the pressure oscillation of pipe network 
turns out to be more and more obvious with the increasing hydrogen blending ratio at transient states. Guandalini et al. [17] analyzed the effect of hydrogen blending on hydraulic conditions of a natural gas pipeline, and found the impact of low hydrogen blending ratio $(5 \%$ volume fraction) on pressure drop $(0.1 \%)$ is negligible, but the mixed hydrogen was able to increase the volume flow rate of the pipeline.

Later, Wang et al. [18] investigated the influence of hydrogen blending on both hydraulic and thermal characteristics of a long-distance pipeline as well as on the performance of a centrifugal compressor. The effect of temperature change on pipeline transportation capacity was taken into account. It was found the pipeline transportation capacity decreases with the increase of temperature, and the volume flow rate of the pipeline increases with the increasing hydrogen blending ratio, indicating the hydrogen blending reduces the pipeline friction resistance. During the joint operation of the pipeline and centrifugal compressor, both the pressure and flow rate corresponding to the operating point were reduced with the increase of hydrogen blending ratio. Hafsi et al. [19, 20] studied the steady and transient flow of natural gas-hydrogen mixture in a simple triangular pipe network by using the isothermal model ignoring the temperature variation. Results demonstrated that the pressure drop at steady state is enhanced by hydrogen blending, and the flow velocity in the pipe increases significantly as well. In addition, the overpressure at transient condition was proportional to the mass fraction of mixed hydrogen. Wu [21] conducted an investigation on the variation of node flow rate and pressure of Chongqing natural gas pipe networks when 10 hydrogen blending sources were considered. It was revealed that the pressure at each node shows a decline trend with the increasing hydrogen blending ratio, and the nodes with larger flow rate suffer a higher pressure drop. Besides, the pressure drop at the node close to the gas source was greater than that far away from the gas source. The research of Uilhoorn [22] demonstrated when the volume flow rate is fixed, the blended hydrogen can alleviate the pressure drop and temperature drop of the gas pipeline, and the compressor power is significantly reduced as well; however, when the energy flow rate is fixed, the pressure drop increases and the temperature drop decreases, as well as the compressor power raises significantly.

To sum up, the previous researches on the hydraulic condition of Hydrogen-Blended Natural Gas (HBNG) pipeline mainly focused on a single pipeline or a single pipe network, lacking of comprehensive consideration covering both the pipeline and pipe network. The study on the influence of hydrogen blending on the performance of centrifugal compressor is still insufficient. In addition, almost all of these researches are based on the isothermal model of pipeline transportation, the effect of temperature change on the hydraulic characteristics of HBNG transportation is rarely considered. At the same time, the impact of hydrogen blending on the thermal condition of natural gas pipeline is still needed to be further clarified. To fill the gap, in this study the influences of hydrogen blending on hydraulic and thermal characteristics of a long-distance natural gas pipeline with the centrifugal compressor and a ringed multi-loop pipe network are systematically investigated. The effects of hydrogen blending ratio on the performance of the centrifugal compressor and the joint operating point of pipeline and compressor are discussed. This study is expected to provide valuable reference for the development of pipeline transportation technology of HBNG.

The structure of this work is organized as follows: Section 2 introduces the mathematical model describing the hydraulic and thermal conditions of natural gas pipelines and pipe networks; Section 3 presents the numerical method for hydraulic and thermal simulations of gas pipeline transportation based on the linearization technique; Section 4 studies the influences of hydrogen blending on the hydraulic and thermal characteristics of a long-distance pipeline and the performance of a centrifugal compressor; Section 5 discusses the impact of hydrogen blending on the hydraulic and thermal characteristics of a ring-shaped multi-loop pipe network; Concluding remarks of this work are summarized in Section 6.

\section{Mathematical model for HBNG pipeline transportation}

The flow and heat transfer of HBNG in pipeline obey "Laws of mass conservation, momentum conservation, and energy conservation". Correspondingly, the hydraulic and thermal governing equations of HBNG pipeline transportation can be expressed as follows [23],

$$
\begin{gathered}
\frac{\partial p}{\partial t}-\left(\frac{\partial p}{\partial T}\right)_{\rho} \frac{\partial T}{\partial t}+\frac{1}{A}\left(\frac{\partial p}{\partial \rho}\right)_{T} \frac{\partial m}{\partial x}=0 \\
\frac{\partial m}{\partial t}+\left[A-\frac{m^{2}}{A \rho^{2}}\left(\frac{\partial p}{\partial \rho}\right)_{T} \frac{\partial p}{\partial x}+\frac{2 m}{A \rho} \frac{\partial m}{\partial x}\right. \\
=-\frac{\lambda}{2} \frac{A m|m|}{d A \rho}-A \rho g \sin \theta+\frac{m^{2}}{A \rho^{2}}\left(\frac{\partial \rho}{\partial T}\right)_{p} \frac{\partial T}{\partial x}, \\
\frac{\partial T}{\partial t}+w \frac{\partial T}{\partial x}=\frac{1}{\rho c_{v}}\left[-T\left(\frac{\partial p}{\partial T}\right)_{\rho} \frac{\partial w}{\partial x}+\frac{\lambda}{2} \frac{\rho|w|^{3}}{D}-\frac{4 K\left(T-T_{g}\right)}{D}\right],
\end{gathered}
$$

where $A$ is the cross-section area of the pipeline, $\mathrm{m}^{2} ; p$ is the pressure, $\mathrm{Pa} ; \rho$ is the gas density, $\mathrm{kg} / \mathrm{m}^{3} ; w$ is the gas velocity, $\mathrm{m} / \mathrm{s} ; \lambda$ is the coefficient of hydraulic friction; $g$ is the gravitational acceleration, $g=9.81 \mathrm{~m} / \mathrm{s}^{2} ; \theta$ is the inclination angle of the pipeline, $\mathrm{rad} ; c_{v}$ is the specific heat capacity at constant volume, $\mathrm{J} /\left(\mathrm{kg} \cdot{ }^{\circ} \mathrm{C}\right) ; K$ is the total heat transfer coefficient of the pipeline, $\mathrm{W} /\left(\mathrm{m}^{2} \cdot{ }^{\circ} \mathrm{C}\right)$; $T$ is the gas temperature, ${ }^{\circ} \mathrm{C} ; T_{g}$ is the temperature of ambient media around the pipeline, ${ }^{\circ} \mathrm{C} ; D$ is the outer diameter of the pipeline, $\mathrm{m}$.

The state parameters ( $P V T$ relationship) of HBNG can be calculated by the gas Equation Of State (EOS), such as the SRK-EOS [24], PR-EOS [25], BWRS-EOS [26], and 
GERG2008-EOS [27], etc. In this study, the widely used BWRS-EOS in natural gas engineering is applied,

$$
\begin{aligned}
p= & \rho R T+\left(B_{0} R T-A_{0}-\frac{C_{0}}{T^{2}}+\frac{D_{0}}{T^{3}}-\frac{E_{0}}{T^{4}}\right) \rho^{2} \\
& +\left(b R T-a-\frac{d}{T}\right) \rho^{3}+\alpha\left(a+\frac{d}{T}\right) \rho^{6} \\
& +\frac{c \rho^{3}}{T}\left(1+\gamma \rho^{2}\right) \exp \left(-\gamma \rho^{2}\right),
\end{aligned}
$$

where $p$ is the gas pressure, $\mathrm{kPa} ; T$ is the gas temperature, $\mathrm{K} ; \rho$ is the gas density, $\mathrm{kmol} / \mathrm{m}^{3} ; R$ is the gas constant, $R=8.3145 \mathrm{~kJ} /(\mathrm{kmol} \cdot \mathrm{K}) . A_{0}, B_{0}, C_{0}, D_{0}, E_{0}, a, b, c, d$, $\alpha$ and $\gamma$ represent the model parameters in BWRS-EOS. For a pure gas component, these 11 parameters can be calculated according to the critical pressure, critical density and acentric factor. For HBNG mixture, the 11 parameters can be calculated based on that of each pure gas component by the mixing rules.

Equations (1)-(4) are the basic mathematical models for the HBNG in pipelines and pipe networks. Compared with the long-distance pipeline, the pipe network is composed of multiple pipelines and nodes. At each junction node, the flow rate, pressure, and outlet temperature should satisfy following relationships,

$$
\begin{gathered}
\sum_{i=1}^{N_{\text {in }}} m_{\text {in }, i}=\sum_{j=1}^{N_{\text {out }}} m_{\text {out }, j}, \\
p_{\text {in }, 1}=\ldots=p_{\text {in }, N_{\text {in }}}=p_{\text {out }, 1}=\ldots=p_{\text {out }, N_{\text {out }}}, \\
T_{\text {out }, 1}=\ldots=T_{\text {out }, N_{\text {out }}}=\sum_{i=1}^{N_{\text {in }}}\left|c_{p} m T\right|_{\text {in }, i} / \sum_{j=1}^{N_{\text {out }}}\left|c_{p} m\right|_{\text {out }, j},
\end{gathered}
$$

where $N$ represents the number of junction nodes; $m$ is the mass flow rate, $\mathrm{kg} / \mathrm{s} ; c_{p}$ is the specific heat capacity at constant pressure, $\mathrm{J} /\left(\mathrm{kg} \cdot{ }^{\circ} \mathrm{C}\right)$; the subscripts "in" and "out" denote the inlet and outlet of junction nodes, respectively.

In a HBNG pipeline system, the centrifugal compressor is an important equipment providing the energy head for transportation, and the fitting of compressor performance curve is needed in the calculation. In this work, the following relations are applied to fit the pressure ratio and polytropic efficiency of a centrifugal compressor,

$$
\begin{gathered}
\varepsilon=A_{\varepsilon} Q^{2}+B_{\varepsilon} Q+C_{\varepsilon}, \\
\eta_{\mathrm{pol}}=A_{\eta} Q^{2}+B_{\eta} Q+C_{\eta},
\end{gathered}
$$

where $n$ denotes the rotational speed, rpm; $A_{\varepsilon}=a_{\varepsilon 1} n^{2}+$ $a_{\varepsilon 2} n+a_{\varepsilon 3}, B_{\varepsilon}=b_{\varepsilon 1} n^{2}+b_{\varepsilon 2} n+b_{\varepsilon 3}, C_{\varepsilon}=c_{\varepsilon 1} n^{2}+c_{\varepsilon 2} n+c_{\varepsilon 3}$, $a_{\varepsilon 1}, a_{\varepsilon 2}, a_{\varepsilon 3}, b_{\varepsilon 1}, b_{\varepsilon 2}, b_{\varepsilon 3}, c_{\varepsilon 1}, c_{\varepsilon 2}$ and $c_{\varepsilon 3}$ are the parameters to be fitted in the pressure ratio curve; $A_{\eta}=a_{\eta 1} n^{2}+$ $a_{\eta 2} n+a_{\eta 3}, \quad B_{\eta}=b_{\eta 1} n^{2}+b_{\eta 2} n+b_{\eta 3}, \quad C_{\eta}=c_{\eta 1} n^{2}+c_{\eta 2} n+$ $c_{\eta 3}, a_{\eta 1}, a_{\eta 2}, a_{\eta 3}, b_{\eta 1}, b_{\eta 2}, b_{\eta 3}, c_{\eta 1}, c_{\eta 2}$ and $c_{\eta 3}$ are the parameters to be determined in the polytropic efficiency curve.

\section{Numerical methods}

The hydraulic and thermal governing equations (1)-(3) are typical nonlinear hyperbolic equations. For the convenience of calculation, first the linearization technique [28] is used to reformulate equations (1)-(3) into the following linear form,

$$
\frac{\partial U}{\partial t}+B(U) \frac{\partial U}{\partial x}=F(U)
$$

where the expression of $U, B(U)$ and $F(U)$ is listed in Table 1, in which the first row denotes the hydraulic equation and the second row stands for the thermal equation.

To discretize the problem in space and time, the spatial step is set as $\Delta x$ and the time step is set as $\Delta t$. In this study, the uniform mesh presented in Figure 1 is employed to discretize the pipeline into $N$ segments, and the nodes are labeled as $1,2, \ldots, N+1$. The nodes 1 and $N+1$ denote the boundary of the pipeline.

In this work, the finite difference method is applied to discretize equation (10) on the mesh shown in Figure 1. Specifically, the unsteady term of hydraulic governing equation is discretized by the forward difference scheme, and the convection term is discretized by the second-order central difference scheme. While for the thermal governing equation, the convection term is discretized by the first-order upwind scheme. Readers of interest can refer to [28] for more detail. The discrete hydraulic governing equation can be arranged as follows,

$$
\boldsymbol{C \boldsymbol { E } _ { i }} \boldsymbol{U}_{i}^{n}+\boldsymbol{D} \boldsymbol{W}_{i} \boldsymbol{U}_{i+1}^{n}=\boldsymbol{H}_{i}
$$

where $\quad C E_{i}=-\frac{1}{2 \Delta t} I-\frac{1}{x_{i+1}-x_{i}} \bar{B}+\frac{1}{2}\left(\bar{G}-\frac{\overline{\partial F}}{\partial U^{T}}\right), \quad D W_{i}=$ $-\frac{1}{2 \Delta t} I-\frac{1}{x_{i+1}-x_{i}} \bar{B}+\frac{1}{2}\left(\bar{G}-\frac{\overline{\partial F}}{\partial U^{T}}\right), H_{i}=\bar{F}+\left[\frac{1}{2}\left(\bar{G}-\frac{\overline{\partial F}}{\partial U^{T}}\right)+\frac{1}{\Delta t}\right] \bar{U}$, and $\boldsymbol{I}$ is a $2 \times 2$ identity matrix, while the matrix $\boldsymbol{G}$ is given by,

$$
\begin{aligned}
& \boldsymbol{G}=\left[\begin{array}{l}
\frac{1}{A} \frac{\partial}{\partial p}\left(\frac{\partial p}{\partial \rho}\right)_{T} \frac{\partial m}{\partial x} \\
\left\{\frac{m^{2}}{A}\left[\frac{2}{\rho^{3}}\left(\frac{\partial \rho}{\partial p}\right)_{T}^{2}-\frac{1}{\rho^{2}}\left(\frac{\partial \rho}{\partial p}\right)_{T}\right] \frac{\partial p}{\partial x}-\frac{2 m}{A \rho^{2}}\left(\frac{\partial \rho}{\partial p}\right)_{T} \frac{\partial m}{\partial x}\right\}
\end{array}\right. \\
& \left.-\frac{2 m}{A \rho^{2}}\left(\frac{\partial \rho}{\partial p}\right)_{T} \frac{\partial p}{\partial x}+\frac{2}{A \rho} \frac{\partial m}{\partial x}\right] .
\end{aligned}
$$

Similarly, the discrete thermal governing equation can be written as below [29],

$$
U P_{i} T_{i-1}^{n}+C E_{i} T_{i}^{n}+D W_{i} T_{i+1}^{n}=H_{i}
$$

where $U P_{i}=-\max \left(w_{i}^{n}, 0\right) \frac{1}{x_{i+1}-x_{i}}, C E_{i}=\frac{1}{\Delta t^{n-1}}-S\left(T_{i}^{n-1}\right)+$ $\left|w_{i}^{n}\right| \frac{1}{x_{i+1}-x_{i}}, \quad D W_{i}=-\max \left(w_{i}^{n}, 0\right) \frac{1}{x_{i+1}-x_{i}}, H_{i}=F\left(T_{i}^{n-1}\right)+$ $\left[\frac{1}{\Delta t}-S\left(T_{i}^{n-1}\right)\right] T_{i}^{n-1}$.

It can be observed that the coefficient matrix of linear equations (11) and (13) is in a Tridiagonal form and is a sparse matrix. Therefore, the Tridiagonal Matrix Algorithm (TDMA) is adopted to efficiently solve the linear systems (11) and (13). Besides, the acceleration techniques 
Table 1. The coefficients of linear equation system.

\begin{tabular}{lcc}
\hline$U$ & $B(U)$ & $F(U)$ \\
\hline$\left[\begin{array}{c}p \\
m\end{array}\right]$ & {$\left[\begin{array}{cc}0 & \frac{1}{A}\left(\frac{\partial p}{\partial \rho}\right)_{T} \\
{\left[A-\frac{m^{2}}{A \rho^{2}}\left(\frac{\partial \rho}{\partial p}\right)_{T}\right]} & \frac{2 m}{A \rho}\end{array}\right]$} & {$\left[\begin{array}{c}\left(\frac{\partial p}{\partial T}\right)_{\rho} \frac{\partial T}{\partial t} \\
\left.-\frac{\lambda}{2} \frac{m|m|}{d A \rho}-A \rho g \sin \theta+\frac{m^{2}}{A \rho^{2}}\left(\frac{\partial \rho}{\partial T}\right)_{p} \frac{\partial T}{\partial x}\right]\end{array}\right.$} \\
$T$ & $w$ & $\frac{1}{\rho c_{v}}\left[-T\left(\frac{\partial p}{\partial T}\right)_{\rho} \frac{\partial w}{\partial x}+\frac{\lambda}{2} \frac{\rho|w|}{D}-\frac{4 K\left(T-T_{g}\right)}{D}\right]$
\end{tabular}

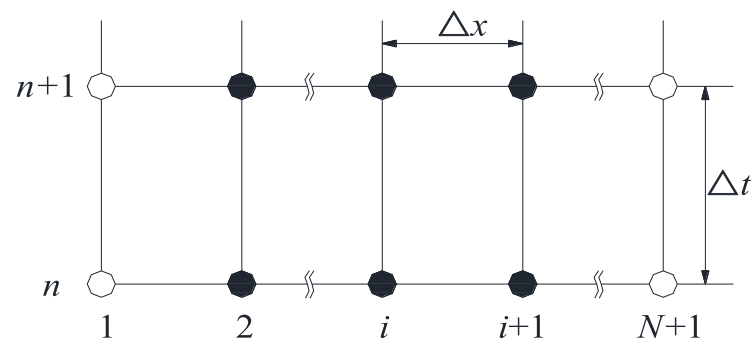

Fig. 1. Discretization in space and time.

can be applied to speed up the solution of linear systems, such as the Graphical-Processing-Unit (GPU)-accelerated algorithm [30].

\section{Effects of hydrogen blending on long- distance natural gas pipeline}

\subsection{Pipeline parameters}

Taking a long-distance pipeline as an example, the influence of hydrogen blending on hydraulic and thermal characteristics of the pipeline transportation system is investigated, such as the flow rate, transportation capacity, outlet pressure, pressure drop, and temperature drop, etc. Besides, the impact of hydrogen blending on the performance of centrifugal compressor is also explored. The detailed parameters and sketch map of the pipeline system are shown in Table 2 and Figure 2, and the composition of natural gas studied in this work is presented in Table 3 .

To quantitatively analyze the influence of hydrogen blending on hydraulic and thermal states of the long-distance pipeline, a proper comparison standard should be established beforehand. Due to the large difference in calorific value between hydrogen and natural gas, mixing hydrogen into natural gas changes the volumetric calorific value of natural gas, thus the volume flow rate cannot truly reflect the gas transmission capacity of the pipeline. Therefore, it is more reasonable to use energy flow rate than volume flow rate to characterize the gas transmission capacity, which is defined as,

$$
\Phi=Q H,
$$

where $\Phi$ is the energy flow rate, $\mathrm{MJ} / \mathrm{h} ; Q$ is the volume flow rate, $\mathrm{m}^{3} / \mathrm{h} ; H$ is the higher calorific value of natural gas mixture, $\mathrm{MJ} / \mathrm{m}^{3}$.
Table 2. Parameters of the long-distance natural gas pipeline.

\begin{tabular}{lc}
\hline Pipeline parameter & Value \\
\hline Size $(\mathrm{mm})$ & $\Phi=1016 \times 17.5$ \\
Transportation capacity $\left(\mathrm{Nm}^{3} / \mathrm{a}\right)$ & $120 \times 10^{8}$ \\
Pipeline length $(\mathrm{km})$ & 183.5 \\
Buried depth $(\mathrm{m})$ & 1.5 \\
Pipeline roughness $(\mu \mathrm{m})$ & 15 \\
Temperature of the gas source $\left({ }^{\circ} \mathrm{C}\right)$ & 34 \\
Maximum soil temperature at the buried & 25.2 \\
depth $\left({ }^{\circ} \mathrm{C}\right)$ & \\
Minimum soil temperature at the buried & 8.4 \\
depth $\left({ }^{\circ} \mathrm{C}\right)$ & \\
Annual average soil temperature at the & 15.0 \\
buried depth $\left({ }^{\circ} \mathrm{C}\right)$ & \\
\hline
\end{tabular}

Demand ○ Node — Pipe

Fig. 2. Sketch map of the studied long-distance pipeline.

Table 3. Composition of natural gas.

\begin{tabular}{lcccccc}
\hline Component & $\mathrm{CH}_{4}$ & $\mathrm{C}_{2} \mathrm{H}_{4}$ & $\mathrm{C}_{3} \mathrm{H}_{8}$ & $\mathrm{~N}_{2}$ & $\mathrm{CO}_{2}$ & $\mathrm{H}_{2}$ \\
\hline Volume fraction (\%) & 97.07 & 0.17 & 0.02 & 0.71 & 2.03 & 0 \\
\hline
\end{tabular}

In hydraulic-thermal simulations, the initial and boundary conditions should be supplemented to close the governing equations. In this study, the following two boundary settings are adopted: (1) control the pressure at the start and the end of the pipeline; (2) control the energy flow rate and the pressure at the end of the pipeline. It is worth pointing out the literature study indicates that when the upper limit of hydrogen blending ratio is $20 \%-30 \%$, the HBNG can be used normally without modifying domestic gas cooking appliances. Thus the concerned hydrogen blending ratio (vol. \%) in this work is set as $0 \%, 5 \%, 10 \%$, $15 \%, 20 \%$ and $30 \%$, respectively. In addition, owning to 
Table 4. Volume flow rate and its deviation of a long-distance pipeline at different hydrogen blending ratios when the pressures at the start and the end of the pipeline are fixed.

\begin{tabular}{|c|c|c|c|c|c|c|}
\hline \multirow[t]{2}{*}{$\mathrm{H}_{2}(\%)$} & \multicolumn{2}{|c|}{$8.4^{\circ} \mathrm{C}$ (winter) } & \multicolumn{2}{|c|}{$15^{\circ} \mathrm{C}$ (average) } & \multicolumn{2}{|c|}{$25.2^{\circ} \mathrm{C}$ (summer) } \\
\hline & $Q\left(10^{7} \mathrm{Nm}^{3} / \mathrm{d}\right)$ & $\varepsilon(\%)$ & $Q\left(10^{7} \mathrm{Nm}^{3} / \mathrm{d}\right)$ & $\varepsilon(\%)$ & $Q\left(10^{7} \mathrm{Nm}^{3} / \mathrm{d}\right)$ & $\varepsilon(\%)$ \\
\hline 0 & 3.86 & 0.00 & 3.838 & 0.00 & 3.808 & 0.00 \\
\hline 5 & 3.91 & 1.28 & 3.888 & 1.29 & 3.858 & 1.32 \\
\hline 10 & 3.96 & 2.76 & 3.945 & 2.79 & 3.916 & 2.84 \\
\hline 15 & 4.03 & 4.46 & 4.011 & 4.51 & 3.983 & 4.58 \\
\hline 20 & 4.10 & 6.39 & 4.086 & 6.45 & 4.058 & 6.55 \\
\hline 30 & 4.28 & 11.03 & 4.265 & 11.13 & 4.238 & 11.27 \\
\hline
\end{tabular}

that the soil temperature at pipeline buried depth is different in different seasons, the temperature of $8.4^{\circ} \mathrm{C}$ in winter, $25.2^{\circ} \mathrm{C}$ in summer, and the annual average temperature of $15^{\circ} \mathrm{C}$, are considered in simulations.

\subsection{Results and discussion}

Firstly, the influence of hydrogen blending on gas transmission capacity of a long-distance pipeline is studied. For the convenience of analysis, the effect of hydrogen blending on compressor performance is discussed in Section 4.3, only the pipeline segment 2-3 in Figure 2 is focused on here. Assuming that the outlet pressure and outlet temperature of the compressor are $9.6 \mathrm{MPa}$ and $50{ }^{\circ} \mathrm{C}$, and the pressure at the pipeline end is $7.4 \mathrm{MPa}$.

Table 4 shows the volume flow rate $(Q)$ of the longdistance pipeline at different hydrogen blending ratios and soil temperatures at pipeline buried depth. It is found if pressures at the start and the end of pipeline 2-3 are fixed, the gas volume flow rate gradually increases with the increasing hydrogen blending ratio whether in winter or in summer. When the hydrogen blending ratio reaches $30 \%$, the volume flow rate of the HBNG pipeline is increased by $11.13 \%$ on average compared with the pipeline without hydrogen, indicating the hydrogen blending has certain influences on gas transmission capacity. The reason can be attributed to properties difference between hydrogen and natural gas, which leads to the reduction of friction resistance along the pipeline with mixed hydrogen, and then the gas volume flow rate increases when the pressures at the start and the end of the pipeline remain unchanged. Table 4 also illustrates that the effect of hydrogen blending on gas volume flow rate of the long-distance pipeline is almost the same under different soil temperatures at pipeline buried depth.

For further discussion, Figure 3a displays the profiles of gas volume flow rate against the hydrogen blending ratio under different soil temperatures at buried depth. It is seen that the three profiles corresponding to summer temperature, winter temperature and annual average temperature are approximately parallel to each other. That is, the influence of hydrogen blending on gas volume flow rate is basically the same in different seasons. It is also found from Figure $3 \mathrm{a}$ that at the same hydrogen blending ratio, the gas volume flow rate in summer is lower than that in winter.
It indicates that with the increase of soil temperature at pipeline buried depth, the gas volume flow rate gradually decreases. It is worth noting that the gas transmission capacity of pipeline segment $2-3$ is not improved although the gas volume flow rate increases with the rise of hydrogen blending ratio when the pressures at the start and the end of pipeline 2-3 are fixed. Figure $3 \mathrm{~b}$ shows the corresponding energy flow rate of the pipeline under the conditions listed in Table 4. Compared with Figures $3 \mathrm{a}$ and $3 \mathrm{~b}$, it is found although the gas volume flow rate increases with the increasing hydrogen blending ratio, the energy flow rate of the pipeline declines almost linearly. The primary reason lies in the fact that under the same condition, the calorific value of hydrogen is about $1 / 3$ of that of natural gas. Thus the mixed hydrogen decreases the calorific value of natural gas, and the increase of volume flow rate is not enough to offset the effect of hydrogen blending on the decrease of calorific value of natural gas.

Figure 4 displays the temperature drop along the pipeline under different soil temperatures at buried depth and different hydrogen blending ratios when the pressures at the start and the end of the pipeline $2-3$ are fixed. It is seen that the variation trend of temperature drop is consistent under different soil temperatures at buried depth. That is, the gas temperature gradually decreases with the increase of pipeline mileage when the gas source temperature remains unchanged. The lower the soil temperature at pipeline buried depth, the greater the total temperature drop along the pipeline, where the temperature drop is the largest in winter and reaches the minimum value in summer. Under the same soil temperature at pipeline buried depth and with the increase of hydrogen blending ratio, the variation trend of temperature drop becomes slower and the total temperature drop of the pipeline decreases gradually. For instance, when the hydrogen blending ratio is $30 \%$, the temperature at the pipeline end is about $2{ }^{\circ} \mathrm{C}$ higher than that of the pipeline without hydrogen. The reason for this phenomenon can be attributed to the decrease of mass flow rate as hydrogen blending ratio increases, which leads to a larger coefficient $a=K \pi D / m c_{p}$ in Sukhov formula for calculating the temperature distribution along the pipeline. In addition, the Joule-Thomson coefficient of natural gas decreases with the increasing hydrogen blending ratio. Compared with the natural gas without hydrogen, the mixed hydrogen has a similar effect with the thermal 
(a)

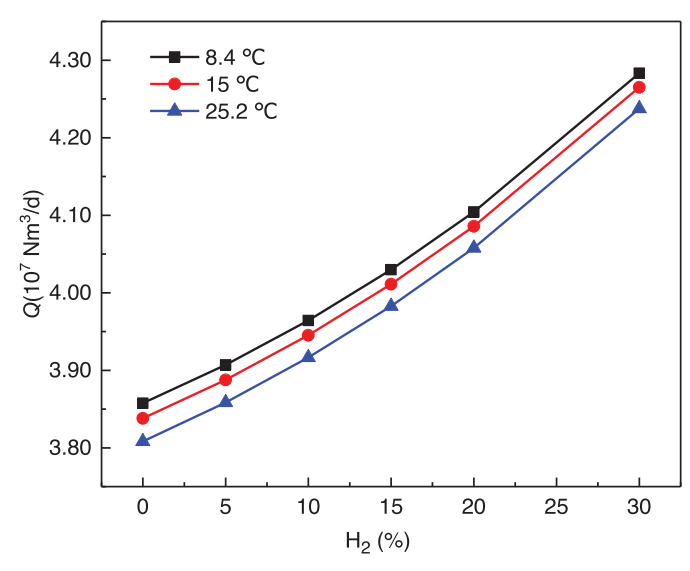

(b)

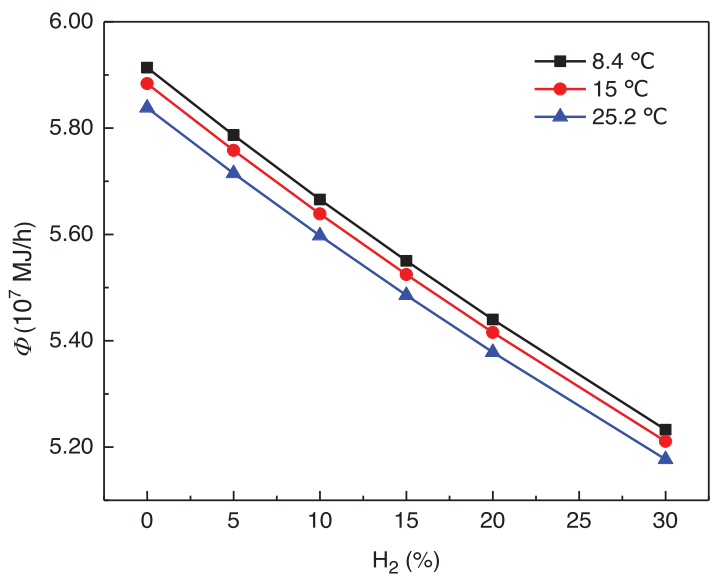

Fig. 3. Variation of volume flow rate and energy flow rate against hydrogen blending ratio under fixed pressures at the start and the end of the pipeline. (a) Volume flow rate; (b) Energy flow rate.

(a)

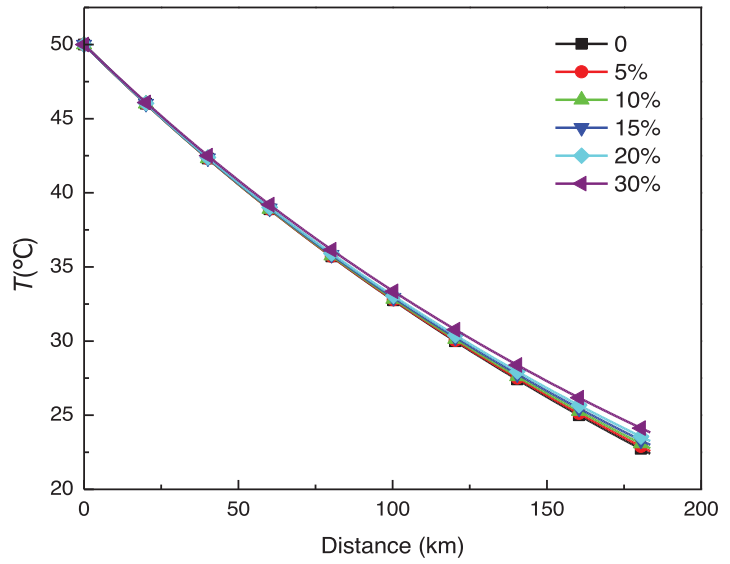

(b)

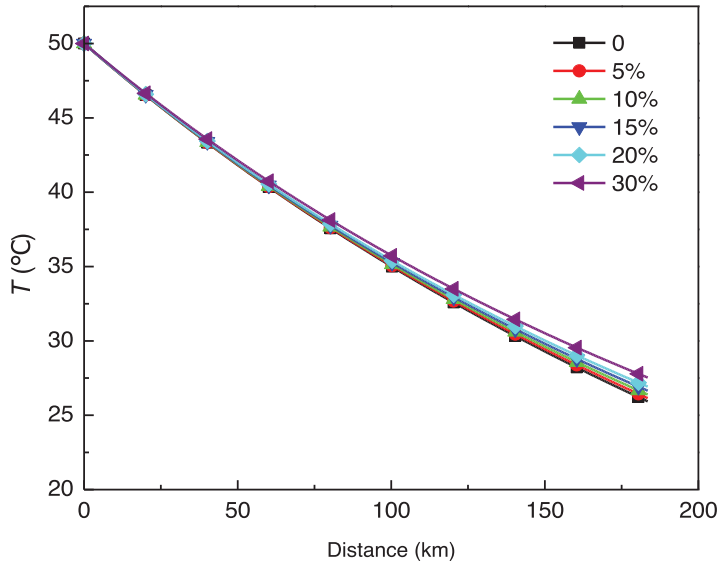

(c)

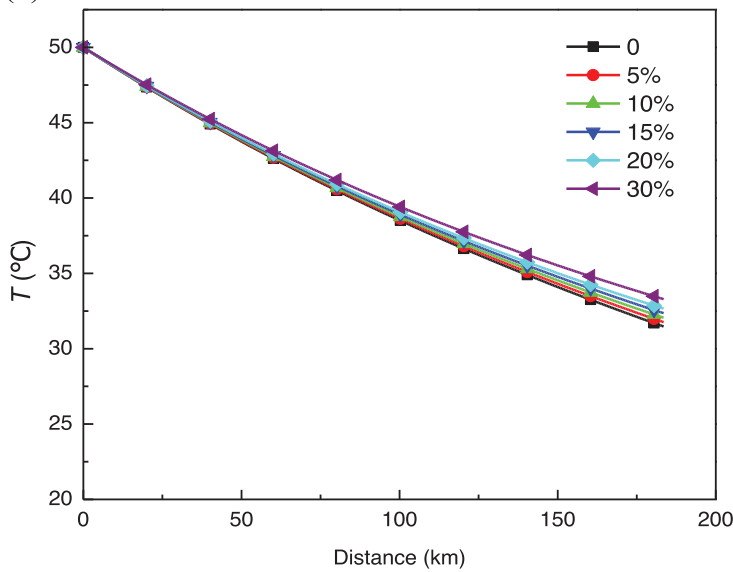

Fig. 4. Temperature drop at different soil temperatures and hydrogen blending ratios when pressures at the start and the end of the pipeline are fixed. (a) Soil temperature is $8.4^{\circ} \mathrm{C}$; (b) Soil temperature is $15{ }^{\circ} \mathrm{C}$; (c) Soil temperature is $25.2{ }^{\circ} \mathrm{C}$.

insulation on the pipeline. The above factors eventually result in that the temperature drop of HBNG is smaller than that of natural gas without hydrogen.
When the energy flow rate and the end pressure of pipeline $2-3$ are fixed, the influence of hydrogen blending on outlet pressure, pressure drop and temperature drop along 
Table 5. Outlet pressure and its deviation of a long-distance pipeline at different hydrogen blending ratios when the energy flow rate and the end pressure of the pipeline are fixed.

\begin{tabular}{|c|c|c|c|c|c|c|c|}
\hline \multirow[t]{2}{*}{$\mathrm{H}_{2}(\%)$} & \multirow[t]{2}{*}{$Q\left(10^{7} \mathrm{Nm}^{3} / \mathrm{d}\right)$} & \multicolumn{2}{|c|}{$8.4^{\circ} \mathrm{C}$ (winter) } & \multicolumn{2}{|c|}{$15^{\circ} \mathrm{C}$ (average) } & \multicolumn{2}{|c|}{$25.2^{\circ} \mathrm{C}$ (summer) } \\
\hline & & $p_{\text {out }}(\mathrm{MPa})$ & $\varepsilon(\%)$ & $p_{\text {out }}(\mathrm{MPa})$ & $\varepsilon(\%)$ & $p_{\text {out }}(\mathrm{MPa})$ & $\varepsilon(\%)$ \\
\hline 0 & 3.84 & 9.58 & 0.00 & 9.60 & 0.00 & 9.63 & 0.00 \\
\hline 5 & 3.97 & 9.67 & 0.89 & 9.68 & 0.88 & 9.71 & 0.88 \\
\hline 10 & 4.12 & 9.75 & 1.78 & 9.77 & 1.78 & 9.80 & 1.76 \\
\hline 15 & 4.27 & 9.84 & 2.69 & 9.86 & 2.68 & 9.89 & 2.66 \\
\hline 20 & 4.44 & 9.93 & 3.60 & 9.94 & 3.59 & 9.97 & 3.56 \\
\hline 30 & 4.82 & 10.10 & 5.46 & 10.12 & 5.43 & 10.15 & 5.39 \\
\hline
\end{tabular}

(a)

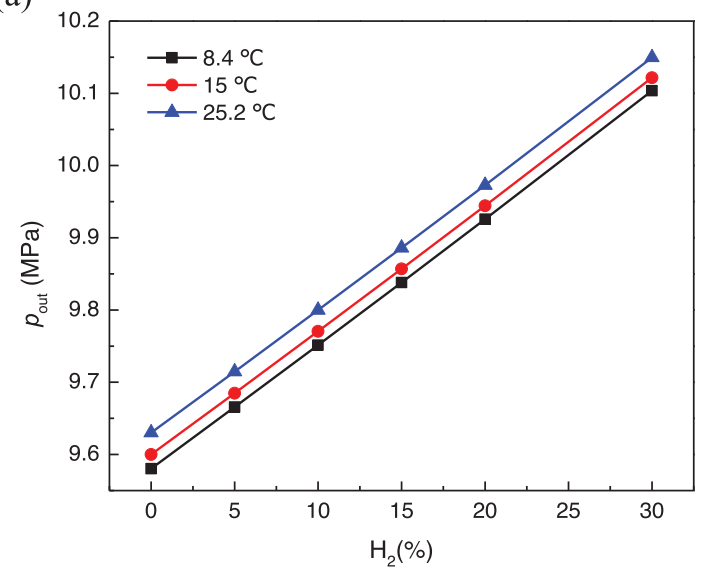

(b)

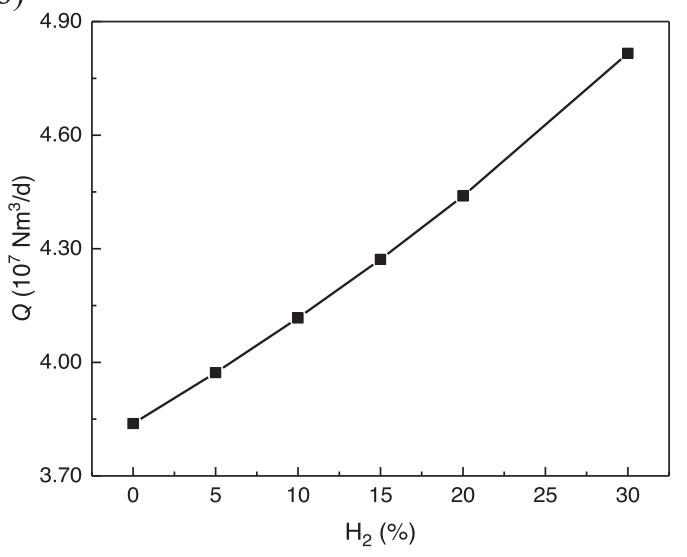

Fig. 5. Variation of outlet pressure and volume flow rate at different hydrogen blending ratios for fixed energy flow rate and end pressure of the pipeline. (a) Outlet pressure; (b) Volume flow rate.

the pipeline is systematically analyzed below. For the sake of simplicity, only the soil temperature of $15^{\circ} \mathrm{C}$ at pipeline buried depth and the corresponding energy flow rate when transporting natural gas without hydrogen are taken for comparison. The temperature at compressor outlet is assumed to be $50{ }^{\circ} \mathrm{C}$.

Table 5 and Figure 5 a demonstrate the outlet pressure of the long-distance pipeline at different hydrogen blending ratios when the energy flow rate and terminal pressure are fixed. It can be seen when the hydrogen blending ratio rises from $0 \%$ to $30 \%$, the outlet pressure of HBNG pipeline is $5.43 \%$ higher than that of traditional natural gas pipeline without hydrogen. The main reason is that for the longdistance pipeline, the adding of hydrogen into the natural gas breaks the original stable operation condition of the pipeline, and the outlet pressure of the compressor will increase to keep the energy flow rate and terminal pressure of the pipeline unchanged. Therefore, it is essential to increase the outlet pressure of the compressor when the energy flow rate and terminal pressure of the pipeline are fixed.

Figure 5a illustrates that the outlet pressure curves corresponding to different soil temperatures at pipeline buried depth have similar variation tendency, indicating the effect of soil temperature on outlet pressure is the same at same hydrogen blending ratio. In addition, since the volumetric calorific value of hydrogen is lower than that of natural gas, when the energy flow rate of pipeline remains unchanged, the volume flow rate of pipeline increases along with the rising hydrogen blending ratio, as shown in Figure 5b. The increased volume flow rate leads to a higher pressure drop along the pipeline. Therefore, when the energy flow rate and terminal pressure of the pipeline are fixed, the pressure drop increases with the raise of hydrogen blending ratio, and thus the outlet pressure of the compressor must be increased, as shown in Figure 6. Similarly, when the energy flow rate and terminal pressure of the pipeline are fixed, the temperature drop declines with the increase of hydrogen blending ratio, as displayed in Figure 7 . The reasons for the effect of hydrogen blending ratio and soil temperature at buried depth on the pressure drop and temperature drop along the pipeline are basically the same, which is not analyzed here for brevity.

\subsection{The influence of hydrogen blending on centrifugal compressor}

Centrifugal compressor is the key equipment in the pipeline transportation system as it provides the required pressure head to overcome the gas flow resistance. When hydrogen 
(a)

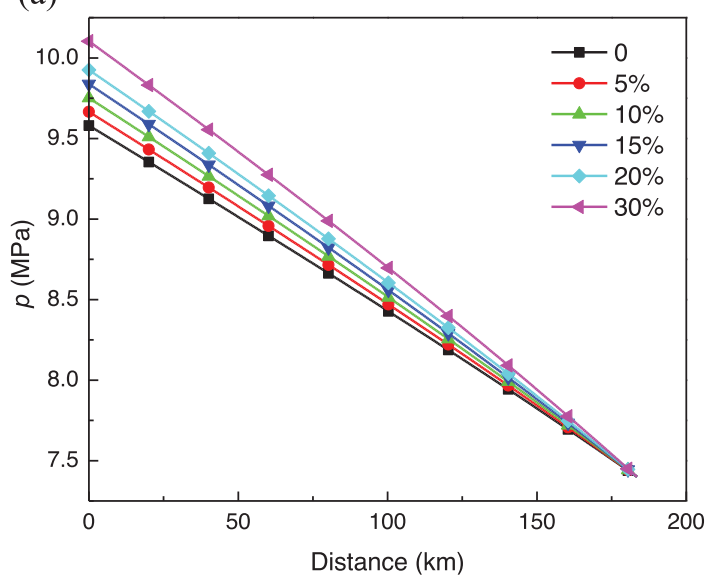

(b)

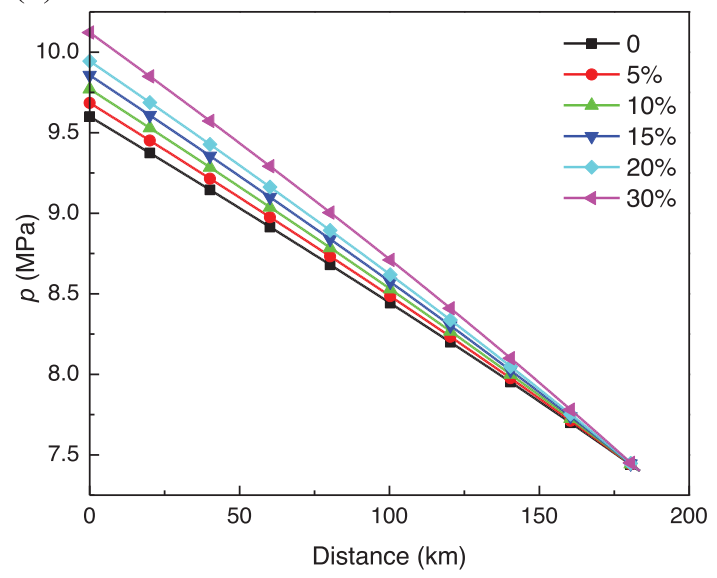

(c)

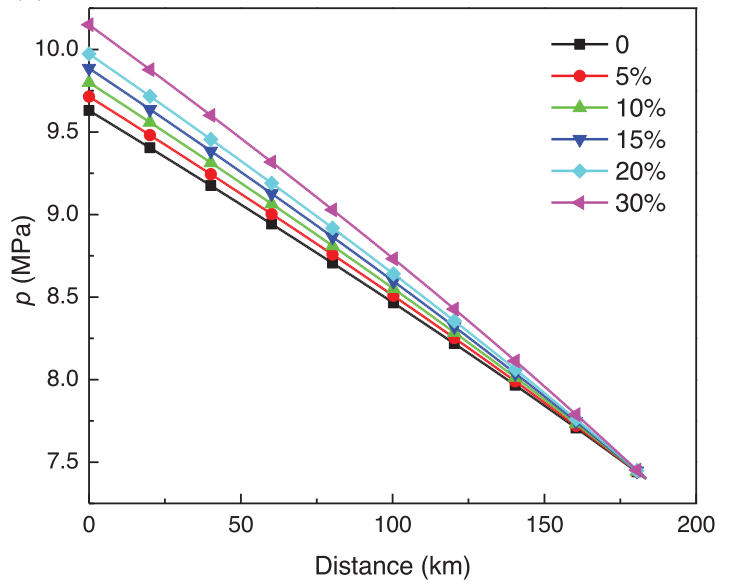

Fig. 6. Pressure drop at different soil temperatures and hydrogen blending ratios for fixed energy flow rate and end pressure of the pipeline. (a) Soil temperature is $8.4^{\circ} \mathrm{C}$; (b) Soil temperature is $15{ }^{\circ} \mathrm{C}$; (c) Soil temperature is $25.2{ }^{\circ} \mathrm{C}$.

is mixed into natural gas, the properties of HBNG are different from that of natural gas without hydrogen, which exerts substantial influences on the performance of centrifugal compressor. Thus it is of great significance to reveal the effect of hydrogen blending on compressor performance. In Table 6, the gas constant and adiabatic index of HBNG at different hydrogen blending ratios are presented. It can be obviously seen that the adiabatic index is weakly affected by the hydrogen blending, with an increase of $2.56 \%$ when the hydrogen blending ratio is $30 \%$. Therefore, the effect of hydrogen blending on the adiabatic index is negligible when the hydrogen blending ratio is small. For the selection of similarity criterion and translation methods of compressor performance curves, literature study [31] found that under the same condition, the translation result of the first kind of similarity method is smaller than the experimental data, while that of the second type of similarity method is larger than the experimental results. However, the relative deviation of the former is much smaller. Hence in this work, the first kind of similarity criterion and translation method is applied to translate the performance curves of centrifugal compressor at different hydrogen blending ratios for convenient comparison.
The centrifugal compressor in Figure 2 takes the PCL-804 compressor as an example. Figures 8 and 9 show the translation curves of shaft power $(W)$ and pressure ratio $\left(p_{r}\right)$ at different hydrogen blending ratios and the rotational speed of $4880 \mathrm{rpm}$ and $5490 \mathrm{rpm}$, respectively. It is seen at two different rotational speeds, the pressure ratio-volume flow rate curve and the shaft power-volume flow rate curve move down with the increasing hydrogen blending ratio. The reason for this phenomenon is that with the increase of hydrogen blending ratio, the density of HBNG decreases, resulting in the increase of gas constant $R_{g}$ and the decrease of pressure ratio. Then the outlet pressure, total energy head and shaft power of the centrifugal compressor decrease as well.

Taking pipeline $1-3$ in Figure 2 as an example, the influences of hydrogen blending on the joint operation of centrifugal compressor and pipeline are explored. Assuming that only the soil temperature of $15{ }^{\circ} \mathrm{C}$ at pipeline buried depth is considered, the volume flow rate is $3.84 \times$ $10^{7} \mathrm{Nm}^{3} / \mathrm{d}$ when the pipeline transmission capacity is fixed, and the gas source temperature and pressure are assumed to be $34{ }^{\circ} \mathrm{C}$ and $6.74 \mathrm{MPa}$. Figure 10 displays the pressure drop and temperature drop when the pipeline is operated 
(a)

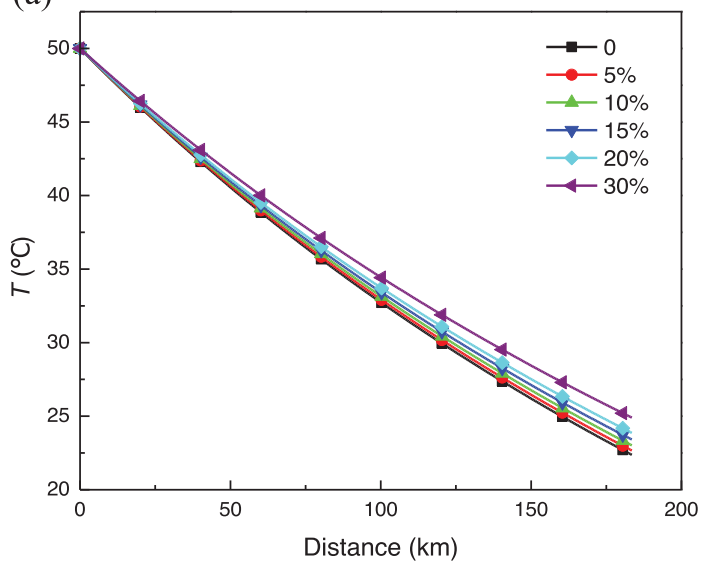

(b)

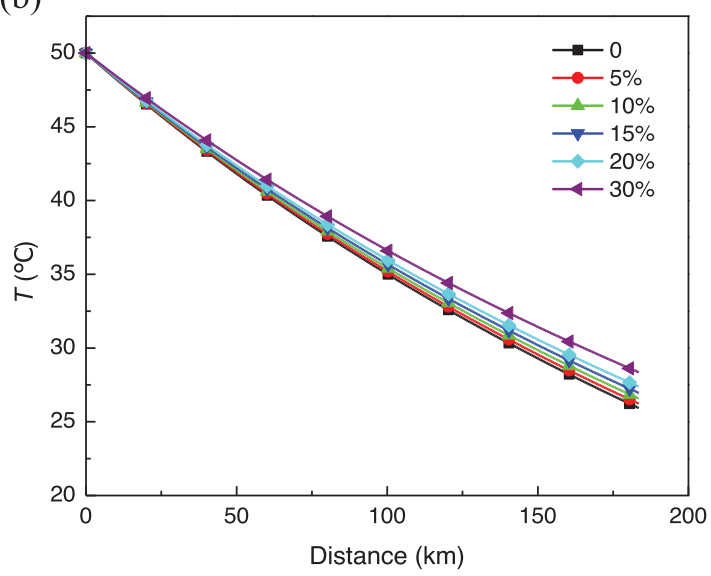

(c)

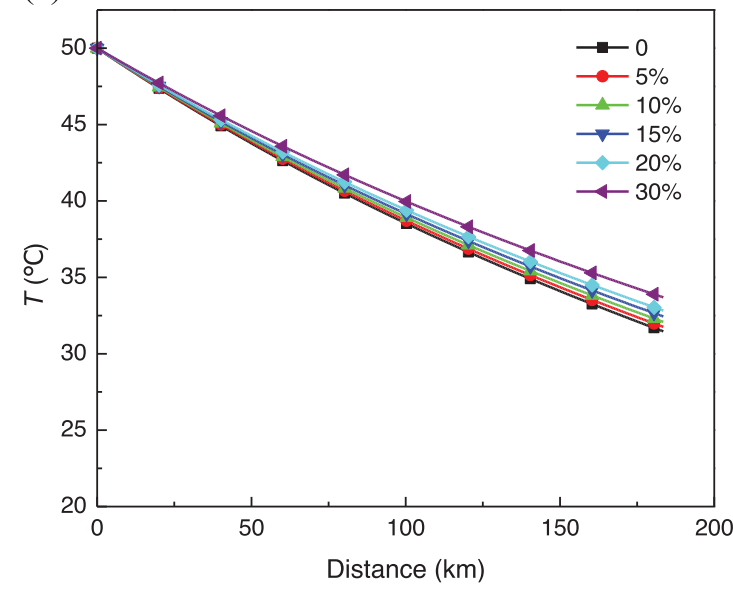

Fig. 7. Temperature drop at different soil temperatures and hydrogen blending ratios for fixed energy flow rate and end pressure of the pipeline. (a) Soil temperature is $8.4^{\circ} \mathrm{C}$; (b) Soil temperature is $15{ }^{\circ} \mathrm{C}$; (c) Soil temperature is $25.2{ }^{\circ} \mathrm{C}$.

Table 6. Gas constant and adiabatic index of natural gas at different hydrogen blending ratios.

\begin{tabular}{lcccccc}
\hline $\mathrm{H}_{2}(\%)$ & 0 & 5 & 10 & 15 & 20 & 30 \\
\hline Gas constant $R_{g}$ & 497.15 & 520.02 & 545.09 & 572.70 & 603.26 & 675.33 \\
Adiabatic index $k$ & 1.29 & 1.30 & 1.30 & 1.31 & 1.31 & 1.32 \\
Relative deviation of $R_{g}(\%)$ & 0 & 4.60 & 9.64 & 15.20 & 21.34 & 35.84 \\
Relative deviation of $k(\%)$ & 0 & 0.43 & 0.85 & 1.28 & 1.71 & 2.56 \\
\hline
\end{tabular}

jointly with the centrifugal compressor. It is found both the outlet pressure and the outlet temperature gradually decrease with the increase of hydrogen blending ratio. Figure 11 shows the joint operation map of the pipeline and centrifugal compressor. It indicates with the increase of hydrogen blending ratio, the characteristic curves of both centrifugal compressor and pipeline move downward, and the operating point moves to the direction of higher volume flow rate and lower pressure. The primary reason for the performance degradation is that the HBNG has a smaller density compared with natural gas without hydrogen, which results in the rise of volume flow rate in the compressor inlet. Then the operating point moves towards the higher volume flow rate and lower pressure at the same time, which ultimately reduces the outlet pressure of centrifugal compressor.

\section{Effects of hydrogen blending on natural gas pipe network}

\subsection{Parameters of pipe network}

In this part, the impacts of hydrogen blending on hydraulic and thermal characteristics of natural gas pipe network are studied by taking the ring-shaped multi-loop pipe network 
(a)

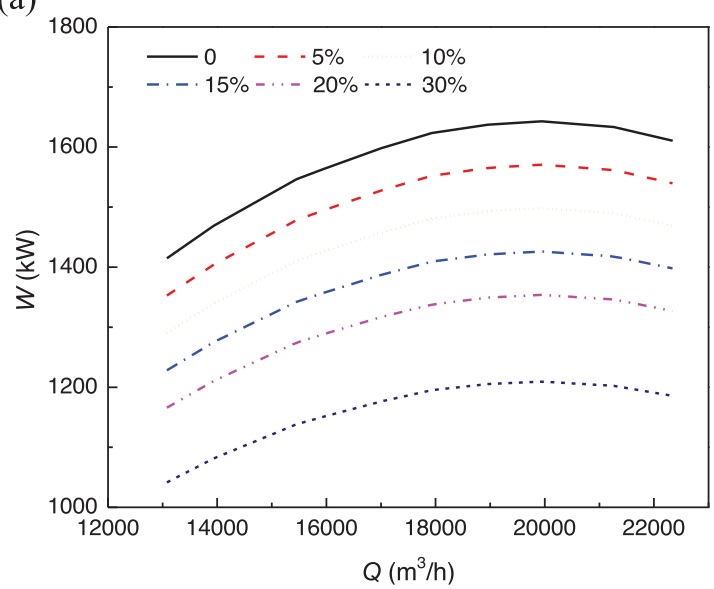

(b)

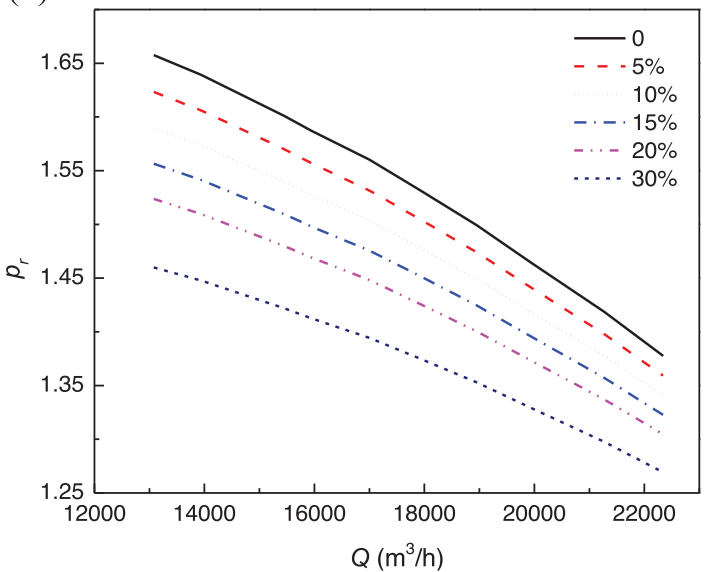

Fig. 8. Performance curve of centrifugal compressor at different hydrogen blending ratios and the rotational speed of $4880 \mathrm{rpm}$. (a) Shaft power; (b) Pressure ratio.
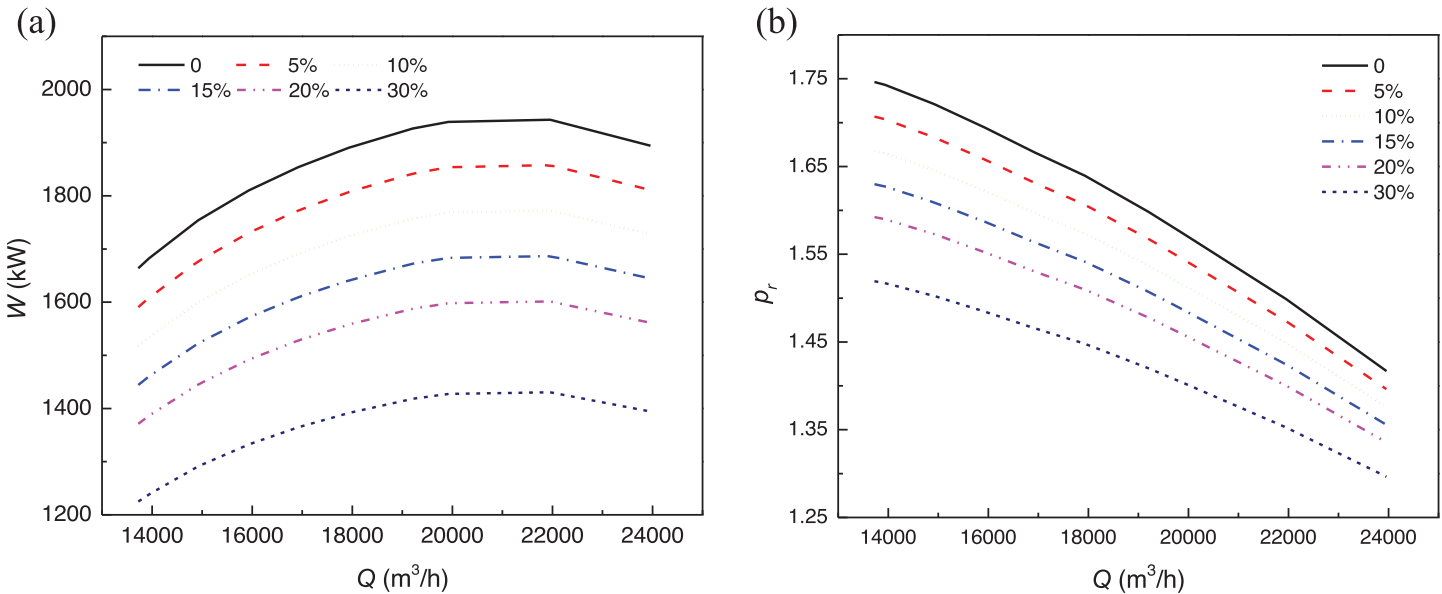

Fig. 9. Performance curve of centrifugal compressor at different hydrogen blending ratios and the rotational speed of $5490 \mathrm{rpm}$. (a) Shaft power; (b) Pressure ratio.

(a)

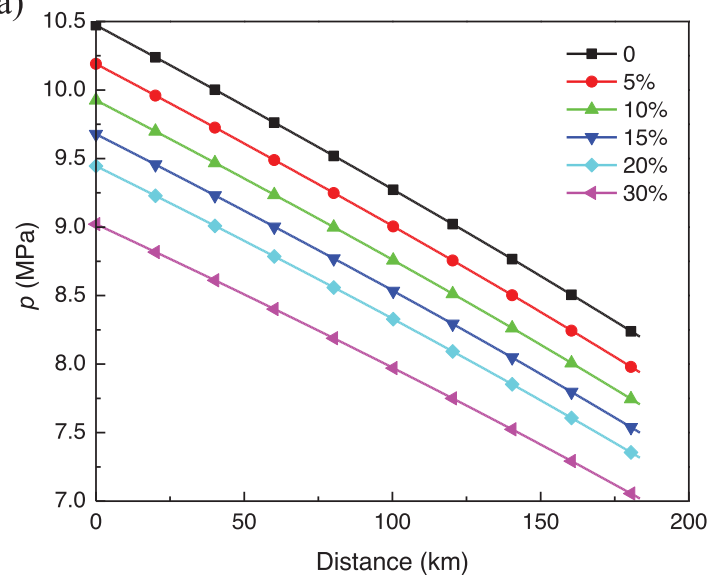

(b)

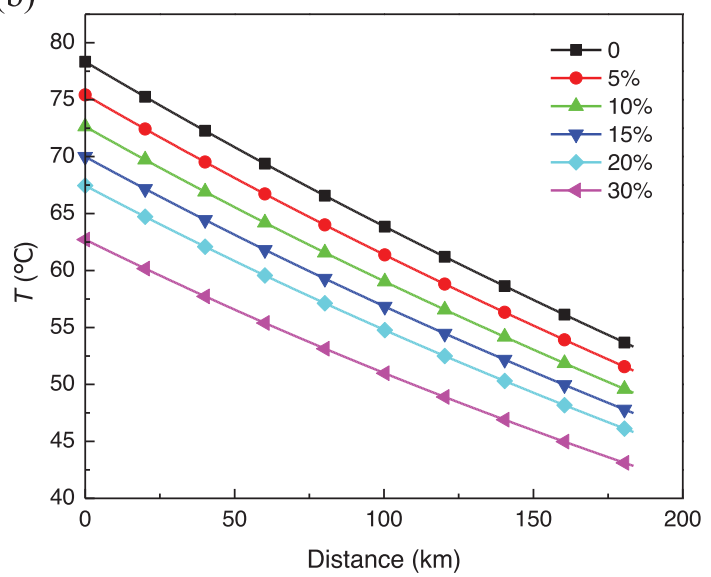

Fig. 10. Pressure and temperature drop for joint operation of pipeline and compressor at $15^{\circ} \mathrm{C}$. (a) pressure drop; (b) temperature drop. 


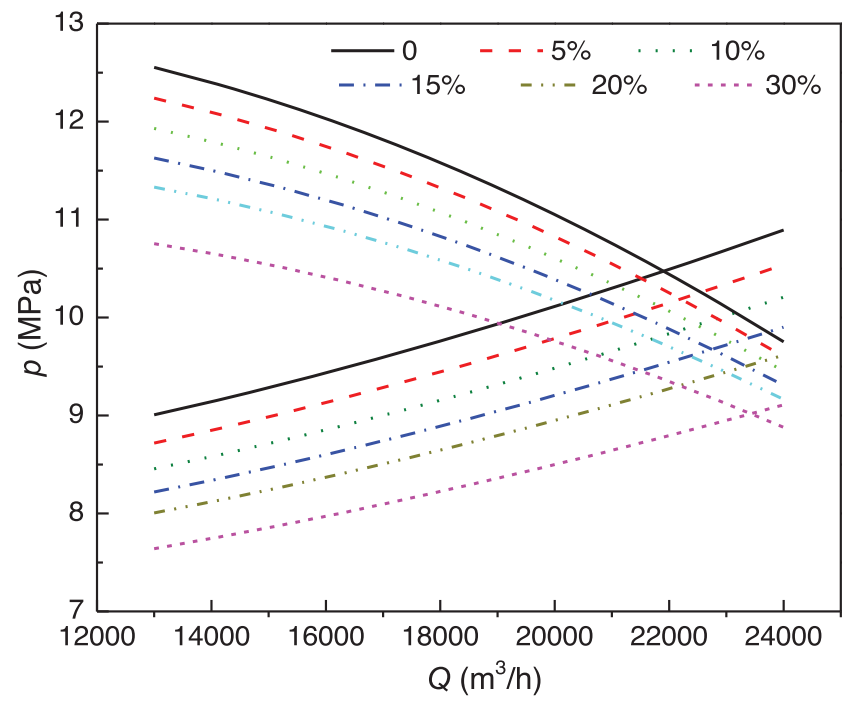

Fig. 11. Performance curve for joint operation of pipeline and compressor.

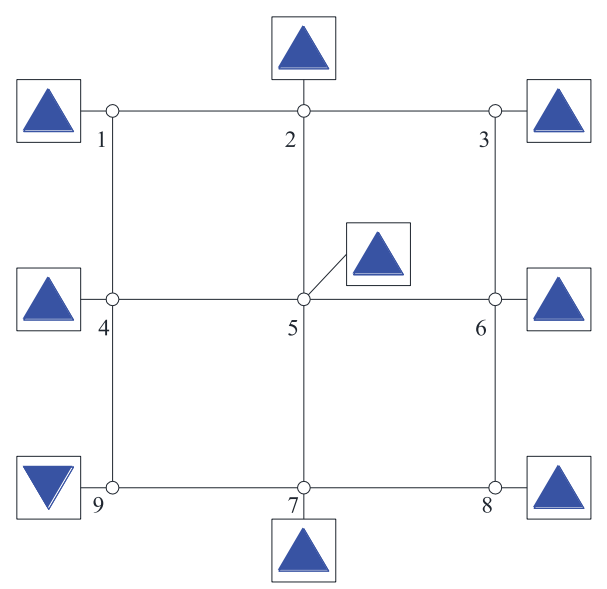

Fig. 12. Topology structure of pipe network.

as an example. The topology of the pipe network is shown in Figure 12, with the pipe parameters and node flow information shown in Tables 7 and 8, where the negative value represents the outflow. The node 9 is the gas supply source, and the pressure of the gas supply source is $360 \mathrm{kPa}$. The composition of natural gas in the pipe network is the same as that in Table 3.

\subsection{Results and discussion}

To investigate the influence of hydrogen blending on hydraulic and thermal characteristics of natural gas pipe network, the gas supply pressure and energy flow rate of the pipe network are kept constant. The variations of pressure and volume flow rate at each node of the pipe network under different hydrogen blending ratios are presented in Table 9 and Figure 13. As can be seen from Table 9 and Figure $13 \mathrm{a}$, with the increase of hydrogen blending ratio, the volume flow rate at each node of the pipe network
Table 7. Parameters of pipe network.

\begin{tabular}{lcccc}
\hline $\begin{array}{l}\text { Pipe } \\
\text { No. }\end{array}$ & $\begin{array}{c}\text { Start } \\
\text { node }\end{array}$ & $\begin{array}{c}\text { End } \\
\text { node }\end{array}$ & $\begin{array}{c}\text { Length } \\
(\mathrm{km})\end{array}$ & $\begin{array}{c}\text { Diameter } \\
(\mathrm{mm})\end{array}$ \\
\hline 1 & 9 & 7 & 1.2 & 147 \\
2 & 7 & 8 & 0.9 & 147 \\
3 & 9 & 4 & 0.8 & 147 \\
4 & 7 & 5 & 0.85 & 125 \\
5 & 8 & 6 & 0.7 & 147 \\
6 & 4 & 5 & 0.7 & 125 \\
7 & 5 & 6 & 0.85 & 147 \\
8 & 4 & 1 & 1 & 125 \\
9 & 5 & 2 & 0.9 & 125 \\
10 & 6 & 3 & 0.8 & 147 \\
11 & 1 & 2 & 1 & 125 \\
12 & 3 & 2 & 0.95 & 147 \\
\hline
\end{tabular}

Table 8. Volume flow rate of each node in the pipe network.

\begin{tabular}{lccc}
\hline Node No. & $Q\left(10^{4} \mathrm{Nm}^{3} / \mathrm{d}\right)$ & Node No. & $Q\left(10^{4} \mathrm{Nm}^{3} / \mathrm{d}\right)$ \\
\hline 1 & -3.60 & 5 & -2.40 \\
2 & -4.32 & 6 & -2.88 \\
3 & -2.40 & 7 & -4.08 \\
4 & -1.92 & 8 & -1.92 \\
\hline
\end{tabular}

increases. When the hydrogen blending ratio reaches $30 \%$, the volume flow rate at each node increases by about $25.48 \%$ compared with that without hydrogen. However, it should be noted although the volume flow rate at each node rises with the increasing hydrogen blending ratio when the gas supply pressure and energy flow rate of the pipe network are fixed, the pressure at each node decreases, as shown in Figure 13b. It is also found the influence of hydrogen blending on the pressure at different nodes varies a lot. That is, under the same hydrogen blending ratio, the pressure drop at the node far away from the gas source (node 9) is significantly larger than that at the node close to the gas source. Figure 13c demonstrates that the temperature drop of the pipe network gradually decreases with the increasing hydrogen blending ratio, showing the same variation trend as that of long-distance pipeline. However, owning to the short distance and low pressure of the pipe network, the temperature variation of pipe network is not obvious, which can be neglected in engineering practice. Thus the natural gas pipe network can be treated as an isothermal model in numerical simulations.

To further explore the impact of hydrogen blending, it is only assumed that the energy flow rate of the pipe network remains constant. Then the variations of pressure, flow rate and temperature at each node under different hydrogen blending ratios are obtained, as shown in Tables 10, 11 and Figure 14. It is seen with the increase of hydrogen blending ratio, most of the pressure at the distribution 
Table 9. Node pressure and volume flow rate under different hydrogen blending ratios at constant gas supply pressure and energy flow rate.

\begin{tabular}{|c|c|c|c|c|c|c|c|c|c|c|}
\hline $\mathrm{H}_{2}(\%)$ & $\backslash$ Node & 1 & 2 & 3 & 4 & 5 & 6 & 7 & 8 & 9 \\
\hline \multirow[t]{2}{*}{0} & $p(\mathrm{kPa})$ & 263.50 & 260.10 & 260.20 & 299.80 & 270.30 & 264.80 & 280.30 & 268.20 & 360.00 \\
\hline & $Q\left(10^{4} \mathrm{Nm}^{3} / \mathrm{d}\right)$ & -3.60 & -4.32 & -2.40 & -1.92 & -2.40 & -2.88 & -4.08 & -1.92 & 23.52 \\
\hline \multirow[t]{2}{*}{5} & $p(\mathrm{kPa})$ & 260.60 & 257.10 & 257.30 & 298.10 & 267.70 & 262.00 & 278.00 & 265.60 & 360.00 \\
\hline & $Q\left(10^{4} \mathrm{Nm}^{3} / \mathrm{d}\right)$ & -3.73 & -4.47 & -2.48 & -1.99 & -2.48 & -2.98 & -4.22 & -1.99 & 24.34 \\
\hline \multirow[t]{2}{*}{10} & $p(\mathrm{kPa})$ & 257.60 & 254.00 & 254.20 & 296.30 & 264.90 & 259.00 & 275.60 & 262.70 & 360.00 \\
\hline & $Q\left(10^{4} \mathrm{Nm}^{3} / \mathrm{d}\right)$ & -3.86 & -4.63 & -2.57 & -2.06 & -2.57 & -3.09 & -4.38 & -2.06 & 25.23 \\
\hline \multirow[t]{2}{*}{15} & $p(\mathrm{kPa})$ & 254.50 & 250.70 & 250.90 & 294.50 & 262.10 & 255.90 & 273.10 & 259.80 & 360.00 \\
\hline & $Q\left(10^{4} \mathrm{Nm}^{3} / \mathrm{d}\right)$ & -4.01 & -4.81 & -2.67 & -2.14 & -2.67 & -3.21 & -4.54 & -2.14 & 26.18 \\
\hline \multirow[t]{2}{*}{20} & $p(\mathrm{kPa})$ & 248.10 & 244.10 & 244.30 & 290.80 & 256.20 & 249.70 & 268.00 & 253.80 & 360.00 \\
\hline & $Q\left(10^{4} \mathrm{Nm}^{3} / \mathrm{d}\right)$ & -4.22 & -5.06 & -2.81 & -2.25 & -2.81 & -3.37 & -4.78 & -2.25 & 27.55 \\
\hline \multirow[t]{2}{*}{30} & $p(\mathrm{kPa})$ & 244.10 & 239.90 & 240.10 & 288.50 & 252.50 & 245.70 & 264.80 & 250.00 & 360.00 \\
\hline & $Q\left(10^{4} \mathrm{Nm}^{3} / \mathrm{d}\right)$ & -4.52 & -5.42 & -3.01 & -2.41 & -3.01 & -3.61 & -5.12 & -2.41 & 29.51 \\
\hline
\end{tabular}

(a)

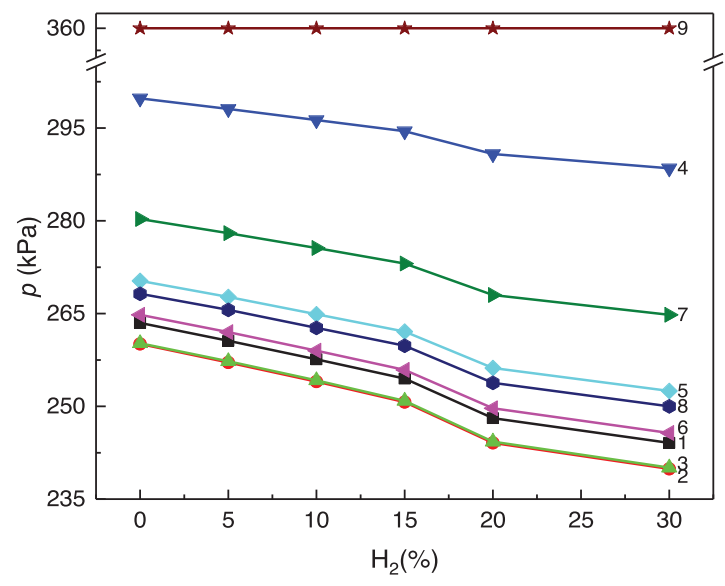

(b)

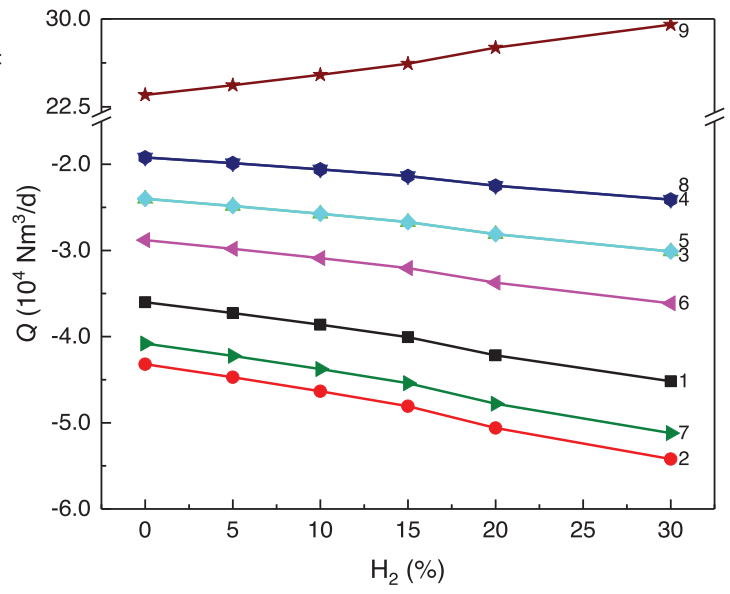

(c)

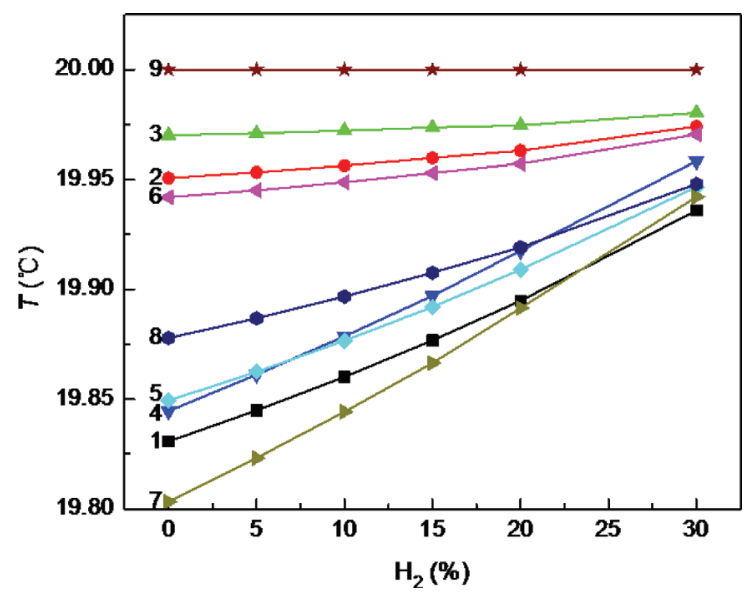

Fig. 13. Variations of node pressure, volume flow rate and gas temperature against hydrogen blending ratio at constant gas supply pressure and energy flow rate. (a) Node pressure; (b) Node volume flow rate; (c) Node temperature. 
Table 10. Node pressure $(\mathrm{kPa})$ under different hydrogen blending ratios at constant energy flow rate.

\begin{tabular}{lccccccccc}
\hline Node $\mathrm{H}_{2}(\%)$ & 1 & 2 & 3 & 4 & 5 & 6 & 7 & 8 & 9 \\
\hline 0 & 243.90 & 240.30 & 240.50 & 282.10 & 251.10 & 245.20 & 261.60 & 248.90 & 344.70 \\
5 & 243.10 & 239.50 & 239.60 & 282.30 & 250.50 & 244.50 & 261.40 & 248.30 & 346.50 \\
10 & 242.60 & 238.80 & 239.00 & 282.90 & 250.20 & 244.10 & 261.30 & 247.90 & 348.50 \\
15 & 242.00 & 238.10 & 238.30 & 283.30 & 249.80 & 243.50 & 261.20 & 247.50 & 350.50 \\
20 & 240.80 & 236.70 & 236.90 & 284.30 & 249.00 & 242.30 & 261.10 & 246.50 & 354.50 \\
30 & 240.10 & 235.80 & 236.10 & 285.00 & 248.60 & 241.70 & 261.10 & 246.10 & 357.00 \\
\hline
\end{tabular}

Table 11. Node volume flow rate $\left(10^{4} \mathrm{Nm}^{3} / \mathrm{d}\right)$ under different hydrogen blending ratios at constant energy flow rate.

\begin{tabular}{llllllllll}
\hline Node $\mathrm{H}_{2}(\%)$ & 1 & \multicolumn{1}{c}{2} & \multicolumn{1}{c}{3} & \multicolumn{1}{c}{4} & \multicolumn{1}{c}{5} & \multicolumn{1}{c}{6} & \multicolumn{1}{c}{7} & \multicolumn{1}{c}{8} & \multicolumn{1}{c}{9} \\
\hline 0 & -3.6 & -4.32 & -2.4 & -1.92 & -2.4 & -2.88 & -4.08 & -1.92 & 23.52 \\
5 & -3.7261 & -4.4713 & -2.4841 & -1.9873 & -2.4841 & -2.9809 & -4.2229 & -1.9873 & 24.3439 \\
10 & -3.8614 & -4.6336 & -2.5742 & -2.0594 & -2.5742 & -3.0891 & -4.3762 & -2.0594 & 25.2275 \\
15 & -4.0068 & -4.8082 & -2.6712 & -2.137 & -2.6712 & -3.2054 & -4.541 & -2.137 & 26.1778 \\
20 & -4.2164 & -5.0597 & -2.811 & -2.2488 & -2.811 & -3.3732 & -4.7786 & -2.2488 & 27.5474 \\
30 & -4.5172 & -5.4207 & -3.0115 & -2.4092 & -3.0115 & -3.6138 & -5.1195 & -2.4092 & 29.5127 \\
\hline
\end{tabular}

(a)

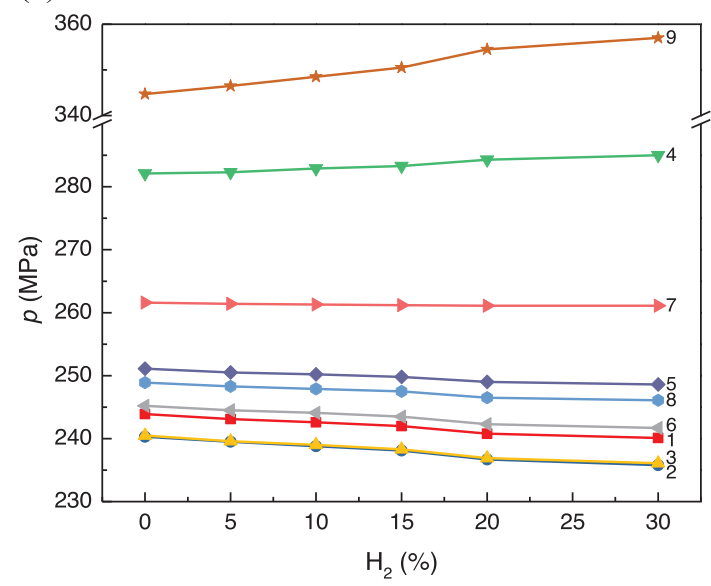

(b)

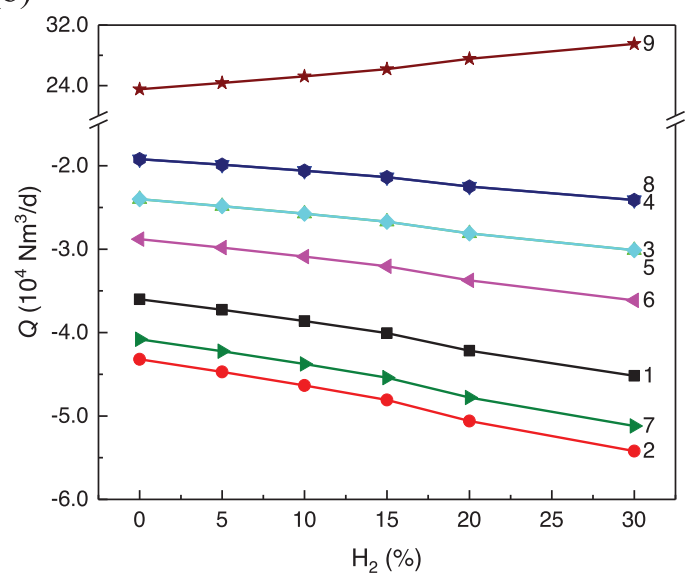

(c)

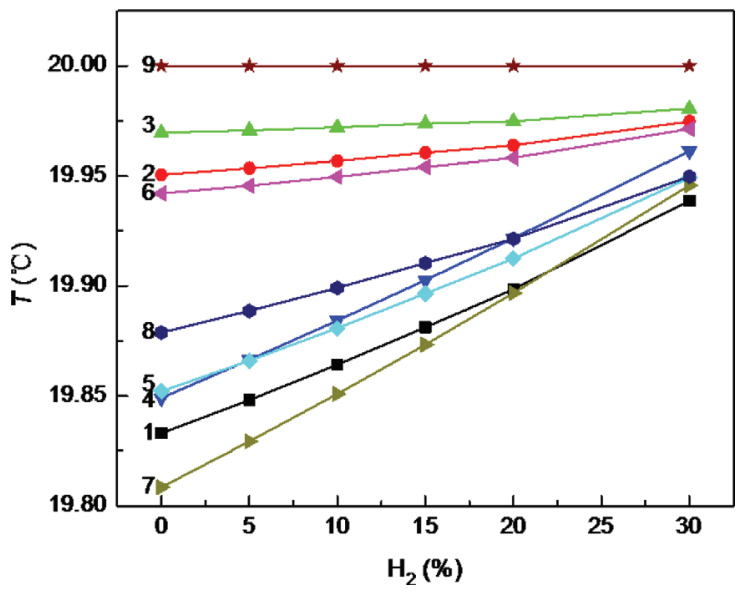

Fig. 14. Variations of node pressure, volume flow rate and gas temperature against hydrogen blending ratios at constant energy flow rate. (a) Node pressure; (b) Node volume flow rate; (c) Node temperature. 
nodes of the pipe network shows a downward trend, while the pressure of the gas supply source and the volume flow rate of all nodes increase. It can be inferred with the increase of hydrogen blending ratio, the larger volume flow rate at each node and higher pressure of gas supply source are required to keep the energy flow rate of the pipe network constant. A similar temperature drop tendency can be observed from Figure 14c, that is, the temperature drop of the pipe network gradually decreases with the increasing hydrogen blending ratio. However, as illustrated above, the temperature change of the pipe network is negligible in engineering practice.

\section{Conclusion}

In this paper, the influences of hydrogen blending on hydraulic and thermal characteristics of a long-distance natural gas pipeline and a ring-shaped multi-loop pipe network are numerically investigated. For the long-distance pipeline, the volume flow rate, energy flow rate, outlet pressure, pressure drop and temperature drop along the pipeline, and performance characteristics of the centrifugal compressor are analyzed at different hydrogen blending ratios. In study of a ring-shaped multi-loop pipe network, the variation trend of node pressure, node volume flow rate, node energy flow rate, and node temperature against the hydrogen blending ratio is comprehensively explored. The main findings of this study are summarized as follows:

1. For the long-distance natural gas pipeline with fixed pressures at the start and the end of the pipeline, the volume flow rate increases with the raise of hydrogen blending ratio, but the energy flow rate shows opposite trend. To keep the energy flow rate constant, the pressure at the compressor outlet should be increased. When the energy flow rate and pressure at the end of the pipeline are fixed, the outlet pressure gradually raises with the increasing hydrogen blending ratio, and the pressure drop along the pipeline increases as well.

2. The temperature drop along the pipeline is negatively related with the soil temperature at pipeline buried depth, that is, the lower the soil temperature, the greater the total temperature drop along the pipeline. Under the same soil temperature, the temperature drop along the pipeline and pipe network slows down with the increase of hydrogen blending ratio, thus the temperature drop becomes smaller for HBNG. Additionally, compared with the temperature drop of gas pipe network which is negligible, the temperature drop along a long-distance pipeline is more significant.

3 . With the increase of hydrogen blending ratio, both the pressure ratio and the shaft power of the centrifugal compressor decrease, resulting in the operating point of the joint operation of compressor and pipeline moves to the direction of higher volume flow rate and lower pressure. Thus it is obvious to know with the increase of hydrogen blending ratio, the rotational speed of centrifugal compressor should be increased to satisfy the transportation requirement at a constant energy flow rate.

4. For the pipe network with fixed gas supply pressure and energy flow rate, the volume flow rate at the nodes increases with the raise of hydrogen blending ratio, while the pressure decreases in this process. At the same hydrogen blending ratio, the pressure drop at the nodes close to the gas supply source is smaller than that of the nodes far away from the gas supply source. When the energy flow rate is fixed, the pressure at the distribution nodes drops and the pressure of gas supply source raises with the increase of hydrogen blending ratio. Meanwhile, the volume flow rate of both distribution nodes and gas supply source increases.

Acknowledgments. This study is supported by the National Natural Science Foundation of China (Nos. 51904031, 51936001), the Natural Science Foundation of Beijing Municipality (No. 3204038), and the Award Cultivation Foundation from Beijing Institute of Petrochemical Technology (No. BIPTACF-002).

\section{Conflict of interest}

The authors declare no competing financial interest.

\section{References}

1 Solomon B.D., Krishna K. (2011) The coming sustainable energy transition: History, strategies, and outlook, Energy Policy 39, 11, 7422-7431.

2 Ye B., Zhang K., Jiang J.J., Miao L.X., Li J. (2017) Towards a $90 \%$ renewable energy future: A case study of an island in the South China Sea, Energ. Convers. Manage. 142, 28-41.

3 Liu B., Liu S.X., Guo S.S., Zhang S.X. (2019) Economic study of a large-scale renewable hydrogen application utilizing surplus renewable energy and natural gas pipeline transportation in China, Int. J. Hydrogen Energ. 45, 3, 1385-1398.

4 Öney F., Veziroğlu T.N., Dülger Z. (1994) Evaluation of pipeline transportation of hydrogen and natural gas mixtures, Int. J. Hydrogen Energ. 19, 10, 813-822.

5 Asadnia M., Mehrpooya M. (2017) A novel hydrogen liquefaction process configuration with combined mixed refrigerant systems, Int. J. Hydrogen Energ. 42, 23, 1556415585 .

6 Adam P., Engelshove S., Heunemann F., Thiemann T., Bussche C.V.D. (2020) Hydrogen infrastructure-the pillar of energy transition: The practical conversion of long-distance gas networks to hydrogen operation, Siemens Energy, Gascade Gastransport GmbH, Nowega GmbH.

7 Haeseldonckx D., D'haeseleer W. (2007) The use of the natural-gas pipeline infrastructure for hydrogen transport in a changing market structure, Int. J. Hydrogen Energ. 32, 10-11, 1381-1386.

8 Yang C., Ogden J. (2007) Determining the lowest-cost hydrogen delivery mode, Int. J. Hydrogen Energ. 32, 2, 268-286. 
9 Messaoudani Z.L., Rigas F., Hamid M.D.B., Hassan C.R.C. (2016) Hazards, safety and knowledge gaps on hydrogen transmission via natural gas grid: A critical review, Int. J. Hydrogen Energ. 41, 39, 17511-17525.

10 International Energy Agency (2019) The future of hydrogen: seizing today's opportunities.

11 Tiekstra G.C., Gasunie N.V.N., Folkert P.K., Gasunie N.V.N. (2008) The NATURALHY project: first step in assessing the potential of the existing natural gas network for hydrogen delivery, IGRC, Paris.

12 Kippers M.J., De Laat J.C., Hermkens R.J.M., Overdiep J.J., Van Der Molen A., Van Erp W.C., Van Der Meer A. (2001) Pilot project on hydrogen injection in natural gas on island of Ameland in the Netherlands, Proc. Int. Gas Research Conf. 2, 1163-1177.

13 Klopffer M.H., Berne P., Espuche É. (2015) Development of innovating materials for distributing mixtures of hydrogen and natural gas. Study of the barrier properties and durability of polymer pipes, Oil Gas Sci. Technol.-Rev. IFP Energies nouvelles 70, 2, 305-315.

14 Tabkhi F., Azzaro-Pantel C., Pibouleau L., Domenech S. (2008) A mathematical framework for modelling and evaluating natural gas pipeline networks under hydrogen injection, Int. J. Hydrogen Energ. 33, 11, 6222-6231.

15 Elaoud S., Hadj-Taïeb E. (2008) Transient flow in pipelines of high-pressure hydrogen-natural gas mixtures, Int. J. Hydrogen Energ. 33, 18, 4824-4832.

16 Elaoud S., Hafsi Z., Hadj-Taïeb L. (2017) Numerical modelling of hydrogen-natural gas mixtures flows in looped networks, J. Petrol. Sci. Eng. 159, 532-541.

17 Guandalini G., Colbertaldo P., Campanari S. (2017) Dynamic modeling of natural gas quality within transport pipelines in presence of hydrogen injections, Appl. Energ. 185, 2, 17121723 .

18 Wang W., Wang Q.Y., Deng H.Q., Cheng G.X., Li Y. (2020) Feasibility analysis on the transportation of hydrogennatural gas mixtures in natural gas pipelines, Nat. Gas Ind. 403, 130-136 (in Chinese).

19 Hafsi Z., Elaoud S., Mishra M. (2019) A computational modelling of natural gas flow in looped network: Effect of upstream hydrogen injection on the structural integrity of gas pipelines, J. Nat. Gas Sci. Eng. 64, 107-117.
20 Hafsi Z., Elaoud S., Akrout M., Hadj-Taïeb E. (2017) Numerical approach for steady state analysis of hydrogennatural gas mixtures flows in looped network, Arab. J. Sci. Eng. 42, 5, 1941-1950.

$21 \mathrm{Wu}$ C. (2018) Feasibility study on blending hydrogen into natural gas distribution network, Chongqing University, Chongqiang, China. (in Chinese).

22 Uilhoorn F. (2009) Dynamic behaviour of non-isothermal compressible natural gases mixed with hydrogen in pipelines, Int. J. Hydrogen Energ. 34, 16, 6722-6729.

23 Li Y.X., Yao G.Z. (2009) Design and management of natural gas pipeline, 2nd edn., Academic Press, Dongying, China (in Chinese).

24 Soave G. (1972) Equilibrium constants from a modified Redlich-Kwong equation of state, Chem. Eng. Sci. 27, 6, 1197-1203.

25 Peng D.Y., Robinson D.B. (1976) A new two-constant equation of state, Ind. Eng. Chem. Fundam. 15, 1, 59-64.

26 Soave G.S. (1999) An effective modification of the BenedictWebb-Rubin equation of state, Fluid Phase Equilibr. 164, 2, 157-172.

27 Varzandeh F., Stenby E.H., Yan W. (2017) Comparison of GERG-2008 and simpler EoS models in calculation of phase equilibrium and physical properties of natural gas related systems, Fluid Phase Equilibr. 434, 21-43.

28 Wang P., Yu B., Deng Y., Zhao Y. (2015) Comparison study on the accuracy and efficiency of the four forms of hydraulic equation of a natural gas pipeline based on linearized solution, J. Nat. Gas Sci. Eng. 22, 235-244.

29 Wang P., Ao S.M., Yu B., Han D.X., Xiang Y. (2019) An efficiently decoupled implicit method for complex natural gas pipeline network simulation, Energies 12, 8, 1516.

30 Xiang Y., Wang P., Yu B., Sun D.L. (2020) GPU-accelerated hydraulic simulations of large-scale natural gas pipeline networks based on a two-level parallel process, Oil Gas Sci. Technol.-Rev. IFP Energies nouvelles 75, 86.

31 Wang L.Y., Wang P., Cao Z.Z., Yu B., Li W. (2017) Similarity conversion of centrifugal natural gas compressors based on predictor-corrector, Procedia Comput. Sci. 108, 1973-1981. 